\title{
Regulating Bacterial Transcription with Small RNAs
}

\author{
G. Storz, * J.A. OpdyKe,* AND K.M. WASSARMAN ${ }^{\dagger}$ \\ *Cell Biology and Metabolism Branch, National Institute of Child Health and Human Development, Bethesda, \\ Maryland 20892-5430; ${ }^{\dagger}$ Department of Bacteriology, University of Wisconsin, Madison, Wisconsin 53706
}

\begin{abstract}
In recent years, the combinations of computational and molecular approaches have led to the identification of an increasing number of small, noncoding RNAs encoded by bacteria and their plasmids and phages. Most of the characterized small RNAs have been shown to operate at a posttranscriptional level, modulating mRNA stability or translation by base-pairing with the $5^{\prime}$ regions of the target mRNAs. However, a subset of small RNAs has been found to regulate transcription. One example is the abundant 6S RNA that has been proposed to compete for DNA binding of RNA polymerase by mimicking the open conformation of promoter DNA. Other small RNAs affect transcription termination via base-pairing interactions with sequences in the mRNA. Here, we discuss current understanding and questions regarding the roles of small RNAs in regulating transcription.
\end{abstract}

The existence of a few small, noncoding RNAs encoded on plasmids, bacteriophage, transposons, and bacterial chromosomes has been known for many decades (generally denoted small RNAs [sRNAs] in bacteria). However, the extensive role these regulatory sRNAs have has only begun to be elucidated in the last few years (for review, see Storz and Gottesman 2006). Most of the sRNAs first identified in bacterial systems were discovered fortuitously. For example, the Escherichia coli sRNAs MicF and DsrA were identified as regulators in multicopy screens. It was then shown that the factor necessary for the observed regulation was an RNA, rather than a protein factor. More recently, systematic approaches, both computational and experimental, have allowed the identification of almost 80 sRNAs encoded on the E. coli chromosome and dozens of sRNA encoded by other bacteria (for review, see Hüttenhofer and Vogel 2006). Several of the computational methods were based on searches for sequence conservation of intergenic regions between a bacterium of interest and closely related organisms. Other computational methods scanned a genome for promoter and stem-loop terminator sequences that did not span known protein-coding regions. Experimental approaches that have led to the identification of sRNAs have been direct cloning of RNAs after size fractionation as well as microarray analysis using total RNA or RNAs that coimmunoprecipitate with known RNA-binding proteins. Each of these approaches has been successful at identifying a subset of sRNAs, but no one approach has been able to identify all known sRNAs, in part due to the high diversity in sRNA conservation, biogenesis, and function.

The sRNAs characterized in $E$. coli thus far fall into three general classes based on their mode of action (for review, see Gottesman 2004; Storz et al. 2005). The first class of RNAs contains intrinsic enzymatic activity such as the rnp RNA that constitutes the catalytic subunit of the RNase P enzyme necessary for processing of pretRNAs. The second class of sRNAs functions by modulating protein activity. In some cases, this class acts by mimicking other nucleic acid structures as is illustrated by the $\mathrm{CsrB} / \mathrm{CsrC}$ sRNAs, which regulate carbon utilization by controlling the activity of the major carbon storage regulator CsrA. A single CsrB RNA molecule contains 18 CsrA-binding sites. Thus, the sRNA is thought to sequester the CsrA protein away from similar binding sites on mRNAs where CsrA binding affects mRNA stability and/or translation. The third and largest class of characterized sRNAs in E. coli is a group of sRNAs that function to regulate target genes through the formation of base-pair interactions with mRNA. At least a third of the E. coli sRNAs are believed to function by this mechanism. More than half of the E. coli sRNAs and even higher percentages of sRNAs in other bacteria remain to be characterized; it will be interesting to see whether other modes of action will be discovered.

sRNAs that act by base-pairing with target mRNAs can be further subclassified into those that are encoded in trans and those RNAs that are encoded in cis relative to their target gene. The trans-encoded sRNAs regulate targets encoded at other locations on the chromosome. The complementarity between trans-encoded sRNAs and their target mRNAs is usually imperfect and limited in length, and many of the sRNAs are capable of regulating more than one target gene. A hallmark of trans-encoded sRNAs is their requirement for the RNA-binding protein Hfq for function; in fact, these sRNAs largely are found in stable complexes with Hfq. Hfq was originally identified as an $E$. coli host factor for $\mathrm{Q} \beta$ phage replication. However, the pleiotropic phenotypes of an $h f q$ mutant suggested a broader role for this protein. It is now recognized that many of the phenotypes of a strain lacking Hfq are due in part to the inability of base-pairing sRNAs to function in this mutant background. The mechanism of Hfq action is not fully understood. In vivo and in vitro studies indicate that Hfq helps facilitate base-pairing between sRNA and target mRNA molecules. In addition, $\mathrm{Hfq}$ is responsible for the high degree of stability of the sRNAs to which it binds; the abundance of many sRNAs is significantly reduced in $h f q$ mutant strains. Further studies of Hfq are necessary to fully understand the complex role of Hfq in sRNA-mediated gene regulation. 
Many of the first sRNAs to be discovered were encoded in cis with their target gene and were found on extrachromosomal DNA molecules such as plasmids, transposons, and bacteriophage, but recently, a small number of cisencoded sRNAs expressed from the $E$. coli chromosome have been identified. By their very nature, cis-encoded RNAs have perfect complementarity with their target RNAs and are thought not to require Hfq for their activity.

The base-pairing between an sRNA and a target mRNA has been shown to have a number of regulatory outcomes at a posttranscriptional level. Many sRNAs can base-pair to mRNAs in regions surrounding the ribosome-binding site and inhibit translation by occluding ribosome access to this recognition sequence. An example is provided by the OxyS RNA, which base-pairs across the ribosomebinding site of $f h l A$ and blocks translation. Base-pairing between sRNAs and mRNAs also has been shown to be associated with the degradation of the target mRNA and possibly the sRNA. For instance, the RyhB RNA is responsible for targeted degradation of a number of mRNAs in a manner that is dependent on the endonuclease RNase E. Although the mRNA degradation may be secondary to the block in translation, the degradation step renders the regulation irreversible. sRNA base-pairing also can activate translation of certain target genes. The $5^{\prime}$ region of the rpoS mRNA folds into a structure that occludes its own ribosome-binding site. Base-pairing between the DsrA RNA with the upstream sequences of rpoS allows alternative folding of the $5^{\prime}$ region into a structure that is accessible to the ribosome.

Although sRNAs that act posttranscriptionally, as described above, have been a focus of many studies during the past 5 years, some of the first sRNAs to be identified were found to modulate transcription, and recent results indicate that the list of sRNAs that act as transcription regulators is likely to continue to expand. Given the numerous components required for accurate transcription as well as the multiple steps involved, it is perhaps not surprising that this step in gene expression would also be subject to regulation by sRNAs. Here, we describe the growing classes of sRNAs that affect the process of transcription and discuss what remains to be learned about these sRNAs.

\section{PROMOTER COMPETITION}

E. coli 6S RNA regulates the first step in transcription, promoter recognition, through direct interaction with the general transcription machinery. Although 6S RNA (184 nucleotides) was one of the first noncoding RNAs to be discovered, its biological function remained a mystery for many years. The finding that this RNA formed a complex with RNA polymerase gave the first hints to its function (Wassarman and Storz 2000). Copurification experiments showed that the 6S RNA forms a stable complex with the housekeeping form of RNA polymerase (denoted $\mathrm{E \sigma}^{70}$ in E. coli), and UV cross-linking studies revealed a direct interaction between the 6S RNA and $\sigma^{70}$ within this complex. Reconstitution experiments confirmed that this interaction is strong and specific (Trotochaud and Wassarman 2005). Thus, the high specificity of 6S RNA for $\mathrm{E}^{70}$ is most likely mediated via the $\sigma^{70}$ subunit.
Phylogenetic analysis of putative 6S RNAs from divergent species revealed that $6 \mathrm{~S}$ is present in a wide range of bacteria and contains a highly conserved secondary structure consisting of a single-stranded central region within a highly double-stranded RNA (Barrick et al. 2005; Trotochaud and Wassarman 2005). The resemblance of this structure to the conformation of DNA within an open complex during transcription initiation led to models in which RNA polymerase interacts with 6S RNA in a manner similar to its usual interactions with promoter DNA. The fact that disruption of this conserved secondary structure by mutation strongly decreases the ability of the 6S RNA to bind $E \sigma^{70}$ suggests the primary mode of recognition of 6S RNA by RNA polymerase is mediated by structural elements, rather than specific sequences (Trotochaud and Wassarman 2005).

6S RNA binding to $\mathrm{E}^{70}$ leads to down-regulation of transcription at several $\sigma^{70}$-dependent promoters in $E$. coli (Trotochaud and Wassarman 2004). However, many promoters are not sensitive to 6S RNA, even late in stationary phase when 6S RNA levels are highest and the vast majority of $\mathrm{E}^{70}$ is bound by $6 \mathrm{~S}$ RNA. In addition to direct effects of $6 \mathrm{~S}$ RNA on $\sigma^{70}$-dependent transcription, indirect regulation of transcription of promoters recognized by an alternative form of RNA polymerase $\left(E \sigma^{\mathrm{S}}\right)$ also has been observed. Thus, it seems likely that $6 \mathrm{~S}$ RNA could be regulating transcription of at least two different forms of RNA polymerases using two modes of action: inhibition of $\mathrm{E \sigma}^{70}$ by substrate competition and activation of $\mathrm{E} \sigma^{\mathrm{S}}$ by sequestration of a potent competitor $\left(E \sigma^{70}\right)$. In any case, it is clear that the formation of stable 6S RNA:E $\sigma^{70}$ complexes leads to altered utilization of $\sigma^{70}$ and $\sigma^{\mathrm{S}}$ during stationary phase. These changes in transcription allow cells to survive starvation and respond to environmental changes when nutrients are limited (Trotochaud and Wassarman 2004, 2006).

A variety of intriguing questions regarding 6S RNA action remain to be addressed. Given that only a subset of $\sigma^{70}$-dependent promoters are affected, what is the nature of the promoter specificity? Does 6S RNA affect the competition between $\sigma^{70}$ and $\sigma^{\mathrm{S}}$ for the rest of the RNA polymerase complex? What contacts are made between 6S RNA and polymerase? Finally, how is the 6S RNA released, and how are the effects of the 6S RNA reversed?

\section{TRANSCRIPTION TERMINATION}

There are several examples of sRNAs that affect transcription termination by base-pairing. The first came from studies of the staphylococcal plasmid pT181 and the inc18 family of streptococcal plasmids (pIP501, pAM $\beta 1$, and pSM19035), which are capable of replicating in a broad host range (Novick et al. 1989; Brantl et al. 1993; Le Chatelier et al. 1996). The replication frequencies of many plasmids are affected by negative feedback by antisense RNAs acting at different levels. In the case of pT181 and the inc18 family of plasmids, this feedback regulation occurs by termination of mRNAs encoding replication proteins. The first system to be characterized was the pT181 plasmid (Novick et al. 1989). For this plas$\mathrm{mid}$, the RepC protein is rate-limiting for replication. Two 
sRNAs, RNAI ( $\sim 85$ nucleotides) and RNAII $(\sim 150$ nucleotides), are encoded on the strand opposite the repC mRNA. Base-pairing between either of the two sRNAs and the repC mRNA results in the formation of a secondary structure that leads to transcription termination (Novick et al. 1989; Brantl and Wagner 2000). In the absence of the base-pairing RNA, a different structure is formed allowing readthrough and complete synthesis of the repC mRNA. The antisense RNAs that modulate expression of replication proteins encoded by the inc 18 family of plasmids are thought to act in a similar fashion (Brantl et al. 1993; Brantl and Wagner 1994).

Another sRNA that likely modulates termination is encoded on the Agrobacterium tumefaciens Ti plasmid (Chai and Winans 2005). Again, replication frequency is regulated by an sRNA, which is encoded opposite replication genes. Increased expression of the RepE RNA ( $\sim 54$ nucleotides) is associated with decreased copy number, whereas decreased RepE expression results in increased copy number. RepE is encoded opposite the repB-repC intergenic sequence, and nuclease S1 protection analysis and assays of repC-lac $Z$ fusions indicated that RepE inhibits RepC expression at the transcriptional level. As in the case for RNAI and RNAII, it is predicted that the RepE base-pairing with the rep $B C$ mRNA causes a secondary structural change, which results in formation of a terminator immediately upstream of repC.

Yet another example is provided by the Vibrio anguillarum virulence plasmid pJM1 (M. Stork et al., in prep.). In this case, the operon encodes four genes for ferricsiderophore transport fatD, fatC, fatB, and fat $A$ and two genes for siderophore biosynthesis, $\operatorname{ang} R$ and $\operatorname{ang} T$. It was observed that the relative level of a transcript encoding the fatDCBA genes is 17 -fold higher than the level of the full-length transcript, and two sRNAs, RNA $\alpha$ and RNA $\beta$ (427 nucleotides), were found to be encoded in cis to the fatDCBAangRT operon. Again, nuclease S1 mapping and lacZ fusion studies suggested that the differential expression of the genes in the operon is due to transcription termination between the fat and angRT genes as a consequence of RNA $\beta$ base-pairing in this region. Exactly how base-pairing might lead to termination remains to be determined.

In the lysogenic state, bacteriophage $\mathrm{P} 4$ prevents the expression of its own replication genes through premature transcription termination. The phage factor responsible for efficient termination is the sRNA CI (79 nucleotides). This RNA is unlike the other examples in that the CI is processed from the leader of the "left-operon" mRNA that it regulates (Briani et al. 2000; Forti et al. 2002). The CI RNA promotes termination at one terminator in the left operon by pairing with a complementary sequence within the leader region.

The structures of the pT181 and pIP501 sRNAs and their target mRNAs as well as their base-pairing interactions have been examined in some detail (Brantl and Wagner 1994, 2000, 2002; Heidrich and Brantl 2003). The formation of the full duplex is not necessary for regulation since base-pairing intermediates preceding the formation of the full duplex are sufficient for full inhibition. It also has been shown that the control is kinetic; the fraction of the target RNA for which termination occurs is determined by the rate of antisense RNA binding. Finally, base-pairing alone appears to be sufficient for the regulation; no requirements for additional protein factors have been observed, and antisense regulation of the pT181 and pIP501 was recapitulated in a heterologous system (Brantl and Wagner 2002). Similarly, RNA $\beta$ of the $V$. anguillarum virulence plasmid and the CI RNA of bacteriophage $\mathrm{P} 4$ were found to enhance termination in the absence of any additional bacterial factor in vitro (Briani et al. 2000; M. Stork et al., in prep.).

All of the sRNAs found to affect transcription termination thus far are encoded on bacterial plasmids or phage, raising the question of whether any chromosomally encoded RNAs act to modulate termination. Although independently transcribed sRNAs that have this activity have not been reported, it should be noted that the $5^{\prime}$-untranslated regions of an increasing number of mRNAs have been found to fold into two alternative structures that in some cases affect termination (for review, see Winkler and Breaker 2005). These so-called riboswitches have been shown to bind a variety of metabolites and even tRNAs; in the presence of the cofactor, they adopt one conformation, whereas a second conformation is adopted in the absence of the cofactor. One structure promotes termination and the other structure permits transcription readthrough.

The examples above illustrate that sRNAs can promote transcription termination and have consequences for the relative expression of genes within an operon. For these basepairing sRNAs, a fair amount is known about the structures of the RNAs and the base-pairing interactions required for regulation. Less is known about the mechanism by which the sRNAs actually affect transcription termination. At what point does base-pairing need to occur in order for termination to occur? Does termination always occur due to the formation of an alternative structure or can duplex formation itself bring about termination as has been shown for oligonucleotides in vitro? In addition, all of the examples lead to termination. One can also imagine that base-pairing could lead to a structure that would prevent the formation of a terminator or block recognition by a termination factor such as the Rho protein, and thus would promote elongation rather than termination.

\section{NONCODING RNA-MEDIATED TRANSCRIPTION REGULATION IN EUKARYOTES}

There has also been an increase in examples of eukaryotic small RNAs (generally denoted noncoding RNAs [ncRNAs] in eukaryotes) that affect transcription (for review, see Goodrich and Kugel 2006). The biggest class is the small interfering RNAs (siRNAs) that are derived from transcripts originating from repeated sequences and act in general silencing of transcription through chromatin remodeling (for review, see Zamore and Haley 2005; Petersen et al. 2006). A different mode of transcriptional regulation is provided by the Saccharomyces cerevisiae SRG1 RNA, which is encoded directly upstream of its regulated gene $S E R 3$. In this case, it is not 
the SRG1 RNA product that is regulatory, but rather the act of transcribing the $S R G 1$ gene that blocks expression of SER3 (Martens et al. 2004, 2005). Other eukaryotic ncRNAs have been shown to affect transcription by binding to specific proteins. Examples of ncRNAs that bind and modify the activities of transcription factors include the NRSE RNA ( 20-bp double-stranded neuronrestrictive silencer element RNA) and HSR1 ( 2000nucleotide heat shock RNA), which modulate the activities of the NRSF/REST repressor and HSF1 transcription factor, respectively (Kuwabara et al. 2004; Shamovsky et al. 2006). The SRA ( 700-nucleotide steroid receptor RNA activator) is thought to act as a coactivator by connecting nuclear hormone receptors to the general transcription machinery (Lanz et al. 1999; Hatchell et al. 2006). The mouse B2 RNA $(\sim 178$ nucleotides), which is transcribed from short interspersed repetitive elements (SINEs), has been shown to inhibit transcription initiation through directing binding to RNA polymerase during the heat shock response (Allen et al. 2004; Espinoza et al. 2004). However, in contrast to the bacterial 6S RNA, the B2 RNA does not block RNA polymerase access to DNA. Instead, the B2 RNA inhibits the initiation of RNA synthesis. The 7SK RNA (331 nucleotides) is unique in that it affects the transition from transcription initiation to elongation by sequestering the P-TEFb elongation factor in a complex with one of its inhibitors, HEX1M1 or HEX1M2 (Nguyen et al. 2001; Yang et al. 2001; Egloff et al. 2006). This inactive form of P-TEFb is not able to phosphorylate the RNA polymerase carboxy-terminal domain (CTD) as is required for promoter escape.

\section{CONCLUSIONS}

The examples described above illustrate some of the different ways in which bacterial sRNAs or eukaryotic ncRNAs can affect transcription. These general mechanisms are summarized in Figure 1. Some RNAs, such as $6 \mathrm{~S}$, NRSE, and B2, modulate the activity of protein complexes by binding to the proteins. Other RNAs, such as SRA and 7SK, appear to act as connectors to bring protein partners together. In contrast, plasmid-encoded RNAs such as RNAI and RepE act by changing the structure of target mRNAs. Whether these examples fully represent all the different ways that the RNAs can act on transcription remains to be seen.

The characterized bacterial sRNAs affect promoter binding and termination. Eukaryotic ncRNAs also have been shown to modulate the activities of transcriptional regulators, inhibit the initiation of RNA synthesis, and affect transcription elongation. It will be interesting to see whether transcription factors and elongation steps are also targeted by sRNAs in bacteria. Similarly, do any eukaryotic ncRNAs base-pair with mRNAs to affect termination, either directly or by altering 3 '-end processing events that could lead to changes in transcription elongation and termination? One can also imagine that sRNAs or ncRNAs might be involved in the coupling of transcription with translation, transcription and RNA processing, or transcription and RNA transport. The spliceosomal U1 snRNA already has been implicated in regulation of transcription (Kwek et al. 2002).

Thousands of cis-encoded antisense RNAs have been reported for eukaryotic organisms. Do any of these RNAs affect transcription? In addition, although most microRNAs (miRNA) are thought to act at the level of regulation of translation and mRNA stability (for review, see ValenciaSanchez et al. 2006), it is conceivable that they could act to create or mask binding sites for RNA-binding proteins involved in other processes as well. When gene regulation was first discovered, it was proposed that the regulators were RNA molecules (Jacob and Monod 1961). This model was superseded by the discovery of protein activators and repressors. The findings described above, however, suggest that the Jacob and Monod model should be revisited. Given that only a fraction of sRNAs and ncRNAs have been characterized, it is likely that many more examples of RNA regulators of transcription as well as unique modes of action remain to be uncovered.
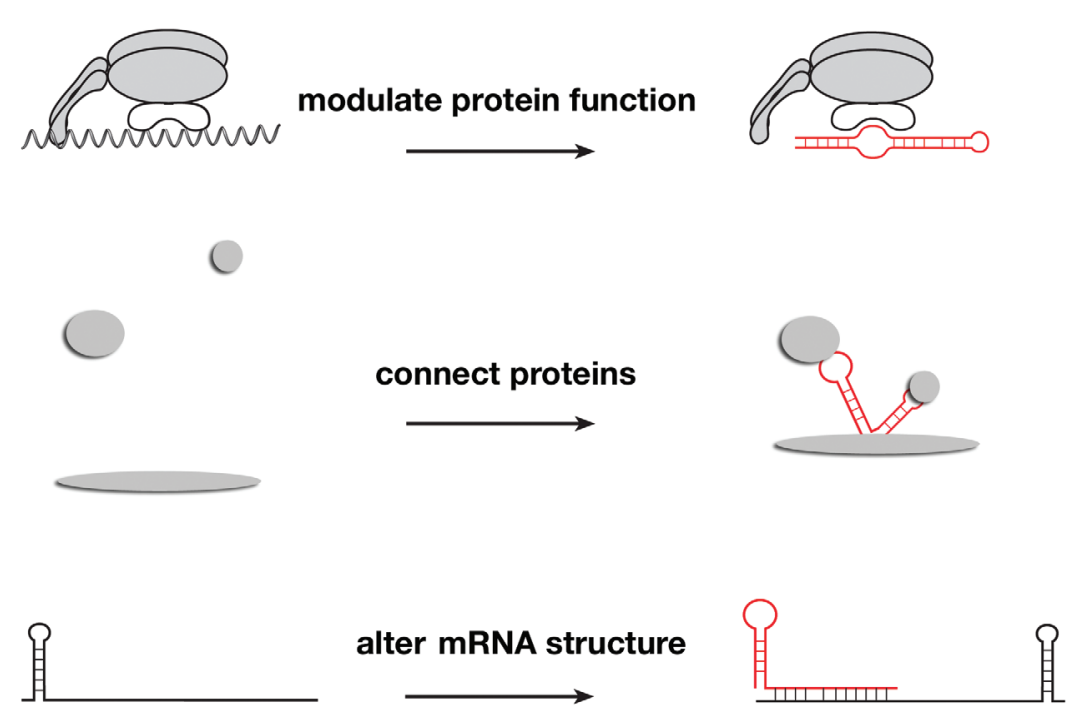

Figure 1. General mechanisms by which RNA regulators (red) modulate transcription. 


\section{ACKNOWLEDGMENTS}

We thank R. Weisberg for comments on the manuscript.

\section{REFERENCES}

Allen T.A., Von Kaenel S., Goodrich J.A., and Kugel J.F. 2004. The SINE-encoded mouse B2 RNA represses mRNA transcription in response to heat shock. Nat. Struct. Mol. Biol. 11: 816.

Barrick J.E., Sudarsan N., Weinberg Z., Ruzzo W.L., and Breaker R.R. 2005. 6S RNA is a widespread regulator of eubacterial RNA polymerase that resembles an open promoter. $R N A$ 11: 774.

Brantl S. and Wagner E.G.H. 1994. Antisense RNA-mediated transcriptional attenuation occurs faster than stable antisense/target RNA pairing: An in vitro study of plasmid pIP501. EMBO J. 13: 3599 .

2000. Antisense RNA-mediated transcriptional attenuation: An in vitro study of plasmid pT181. Mol. Microbiol. 35: 1469.

- 2002. An antisense RNA-mediated transcription attenuation mechanism functions in Escherichia coli. J. Bacteriol. 184: 2740.

Brantl S., Birch-Hirschfeld E., and Behnke D. 1993. RepR protein expression on plasmid pIP501 is controlled by an antisense RNA-mediated transcription attenuation mechanism. J. Bacteriol. 175: 4052 .

Briani F., Ghisotti D., and Dehò G. 2000. Antisense RNA-dependent transcription termination sites that modulate lysogenic development of satellite phage P4. Mol. Microbiol. 36: 1124.

Chai Y. and Winans S.C. 2005. A small antisense RNA downregulates expression of an essential replicase protein of an Agrobacterium tumefaciens Ti plasmid. Mol. Microbiol. 56: 1574.

Egloff S., Van Herreweghe E., and Kiss T. 2006. Regulation of polymerase II transcription by 7SK snRNA: Two distinct RNA elements direct P-TEFb and HEXIM1 binding. Mol. Cell Biol. 26: 630

Espinoza C.A., Allen T.A., Hieb A.R., Kugel J.F., and Goodrich J.A. 2004. B2 RNA binds directly to RNA polymerase II to repress transcript synthesis. Nat. Struct. Mol. Biol. 11: 822

Forti F., Dragoni I., Briani F., Dehò G., and Ghisotti D. 2002. Characterization of the small antisense CI RNA that regulates bacteriophage P4 immunity. J. Mol. Biol. 315: 541.

Goodrich J.A. and Kugel J.F. 2006. Non-coding-RNA regulators of RNA polymerase II transcription. Nat. Rev. Mol. Cell Biol. 7: 612 .

Gottesman S. 2004. The small RNA regulators of Escherichia coli: Roles and mechanisms. Annu. Rev. Microbiol. 58: 303.

Hatchell E.C., Colley S.M., Beveridge D.J., Epis M.R., Stuart L.M., Giles K.M., Redfern A.D., Miles L.E., Barker A., MacDonald L.M., et al. 2006. SLIRP, a small SRA binding protein, is a nuclear receptor corepressor. Mol. Cell 22: 657.

Heidrich N. and Brantl S. 2003. Antisense-RNA mediated transcriptional attenuation: Importance of a U-turn loop structure in the target RNA of plasmid pIP501 for efficient inhibition by the antisense RNA. J. Mol. Biol. 333: 917.

Hüttenhofer A. and Vogel J. 2006. Experimental approaches to identify non-coding RNAs. Nucleic Acids Res. 34: 635.
Jacob F. and Monod J. 1961. Genetic regulatory mechanisms in the synthesis of proteins. J. Mol. Biol. 3: 318 .

Kuwabara T., Hsieh J., Nakashima K., Taira K., and Gage F.H. 2004. A small modulatory dsRNA specifies the fate of adult neural stem cells. Cell 116: 779 .

Kwek K.Y., Murphy S., Furger A., Thomas B., O'Gorman W., Kimura H., Proudfoot N.J., and Akoulitchev A. 2002. U1 snRNA associates with TFIIH and regulates transcriptional initiation. Nat. Struct. Biol. 9: 800.

Lanz R.B., McKenna N.J., Onate S.A., Albrecht U., Wong J., Tsai S.Y., Tsai M.J., and O’Malley B.W. 1999. A steroid receptor coactivator, SRA, functions as an RNA and is present in an SRC-1 complex. Cell 97: 17.

Le Chatelier E., Ehrlich S.D., and Janniere L. 1996. Counter transcript-driven attenuation system of the pAM $\beta 1$ repE gene. Mol. Microbiol. 20: 1099.

Martens J.A., Laprade L., and Winston F. 2004. Intergenic transcription is required to repress the Saccharomyces cerevisiae SER3 gene. Nature 429: 571 .

Martens J.A., Wu P.Y., and Winston F. 2005. Regulation of an intergenic transcript controls adjacent gene transcription in Saccharomyces cerevisiae. Genes Dev. 19: 2695.

Novick R.P., Iordanescu S., Projan S.J., Kornblum J., and Edelman I. 1989. pT181 plasmid replication is regulated by a countertranscript driven transcriptional attenuator. Cell 59: 395.

Nguyen V.T., Kiss T., Michels A.A., and Bensaude O. 2001. 7SK small nuclear RNA binds to and inhibits the activity of CDK9/cyclin T complexes. Nature 414: 322.

Petersen C.P., Doench J.G., Grishok A., and Sharp P.A. 2006. The biology of short RNAs. In The RNA world, 3rd edition (ed. R.F. Gesteland et al.), p. 535. Cold Spring Harbor Laboratory Press, Cold Spring Harbor, New York.

Shamovsky I., Ivannikov M., Kandel E.S., Gershon D., and Nudler E. 2006. RNA-mediated response to heat shock in mammalian cells. Nature 440: 556.

Storz G. and Gottesman S. 2006. Versatile roles of small RNA regulators in bacteria. In The RNA world, 3rd edition (ed. R.F. Gesteland et al.), p. 567. Cold Spring Harbor Laboratory Press, Cold Spring Harbor, New York.

Storz G., Altuvia S., and Wassarman K.M. 2005. An abundance of RNA regulators. Annu. Rev. Biochem. 74: 199.

Trotochaud A.E. and Wassarman K.M. 2004. 6S RNA function enhances long-term cell survival. J. Bacteriol. 186: 4978.

. 2005. A highly conserved 6S RNA structure is required for regulation of transcription. Nat. Struct. Mol. Biol. 12: 313 .

. 2006. 6S RNA regulation of $\mathrm{pspF}$ transcription leads to altered cell survival at high pH. J. Bacteriol. 188: 3936.

Valencia-Sanchez M.A., Liu J., Hannon G.J., and Parker R. 2006. Control of translation and mRNA degradation by miRNAs and siRNAs. Genes Dev. 20: 515.

Wassarman K.M. and Storz G. 2000. 6S RNA regulates E. coli RNA polymerase activity. Cell 101: 613 .

Winkler W.C. and Breaker R.R. 2005. Regulation of bacterial gene expression by riboswitches. Annu. Rev. Microbiol. 59: 487.

Yang Z., Zhu Q., Luo K., and Zhou Q. 2001. The 7SK small nuclear RNA inhibits the CDK9/cyclin T1 kinase to control transcription. Nature 414: 317.

Zamore P.D. and Haley B. 2005. Ribo-gnome: The big world of small RNAs. Science 309: 1519. 


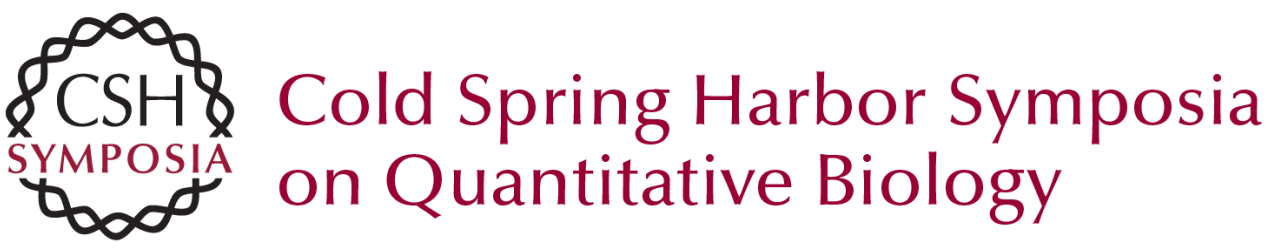

\section{Regulating Bacterial Transcription with Small RNAs}

G. STORZ, J.A. OPDYKE and K.M. WASSARMAN

Cold Spring Harb Symp Quant Biol 2006 71: 269-273

Access the most recent version at doi:10.1101/sqb.2006.71.033

References This article cites 35 articles, 9 of which can be accessed free at:

http://symposium.cshlp.org/content/71/269.full.html\#ref-list-1

\section{License}

Email Alerting Receive free email alerts when new articles cite this article - sign up in Service the box at the top right corner of the article or click here. 